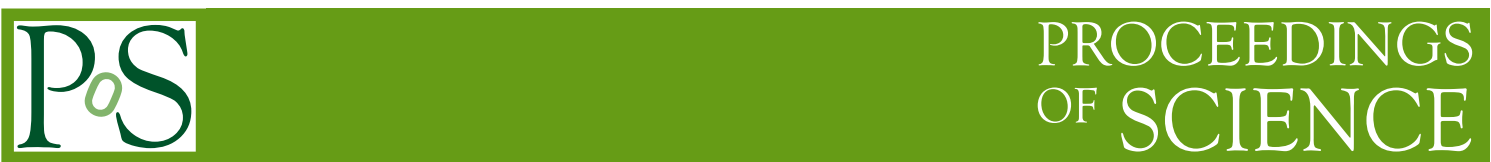

\title{
Explaining the SM flavor structure with grand unified theories
}

\section{Renato Fonseca}

Institute of Particle and Nuclear Physics

Faculty of Mathematics and Physics, Charles University,

V Holešovičkách 2, 18000 Prague 8, Czech Republic

E-mail: fonseca@ipnp.mff.cuni.cz

We do not know why there are three fermion families in the Standard Model (SM), nor can we explain the observed pattern of fermion masses and mixing angles. Standard grand unified theories based on the $S U(5)$ and $S O(10)$ groups fail to shed light on this issue, since they also contain three copies of fermion representations of an enlarged gauge group. However, it does not need to be so: the Standard Model families might be distributed over distinct representations of a grand unified model, in which case the gauge symmetry itself might discriminate the various families and explain (at least partially) the flavor puzzle. The most ambitious version of this idea consists on embedding all SM fermions in a single irreducible representation of the gauge group.

40th International Conference on High Energy physics - ICHEP2020

July 28 - August 6, 2020

Prague, Czech Republic (virtual meeting) 


\section{Flavor and grand unification}

The Standard Model of particle physics is a gauge theory built around the group $S U(3) \times S U(2) \times$ $U(1) \equiv G_{S M}$, with the fermions distributed over 15 irreducible representations: $3\left(Q+u^{c}+d^{c}+\right.$ $\left.L+e^{c}\right)$. The quantum numbers $Q=(\mathbf{3}, \mathbf{2}, 1 / 6), u^{c}=(\overline{\mathbf{3}}, \mathbf{1},-2 / 3), d^{c}=(\overline{\mathbf{3}}, \mathbf{1}, 1 / 3), L=(\mathbf{1}, \mathbf{2},-1 / 2)$ and $e^{c}=(\mathbf{1}, \mathbf{1}, 1)$ are somewhat curious; for example, only the fundamental and anti-fundamental representations of the special unitary groups are used, plus it is possible to write all hypercharges as rational numbers. Grand unified theories (GUTs) provide an elegant explanation for these quantum numbers. The idea is that the true gauge symmetry of Nature is given by a group $G$ larger than $G_{S M}$, which is spontaneously broken at very high energies. If $G$ is a simple group - such as $S U(5)$, $S O(10)$ or $E_{6}[1-4]$ - it then becomes possible to relate the three Standard Model gauge coupling constants. Furthermore, the quantum numbers of fermions under $G_{S M}$ follow directly from the transformation properties of these fields under the enlarged group $G$.

Given that grand unified theories can account for the quantum numbers of the Standard Model fermions in an appealing way, we are left with what seems to be a deeper mystery of the Standard Model, namely the existence of three copies of every irreducible fermion representation $X$ of $G_{S M}$ ( $X=Q, u^{c}, d^{c}, L$ and $e^{c}$ ). Each copy is often called a family, a generation or a flavor. Assigning a flavor index to each fermion, Yukawa interactions are controlled by $3 \times 3$ matrices which account for the measured fermion masses and mixing parameters:

$$
\left(Y_{U}\right)_{i j} Q_{i} u_{j}^{c} H+\left(Y_{D}\right)_{i j} Q_{i} d_{j}^{c} H^{*}+\left(Y_{E}\right)_{i j} L_{i} e_{j}^{c} H^{*}
$$

It is worth noting that in standard GUTs - based on the groups mentioned earlier - the fermion representations also have flavor indices and therefore at high energies the Yukawa couplings can still be seen as matrices in flavor space.

We also do not know why these matrices have the values that they do, but perhaps once we have an explanation for the existence of three families that might become clear. One possibility is that the Standard Model family replication is an accident in the following sense: under a more fundamental gauge group, fermions might be assigned to a combination of representations $R+R^{\prime}+\cdots$ which do not have a trifold repetition. This is in fact what happens with several (but not all) models based on the semi-simple group $S U(3) \times S U(3) \times U(1)$ [5-8]. However, it can also happen with simple groups. A particularly interesting example is the $S U(11)$ model $[9,10]$ where fermions are assigned to the representations $\overline{\mathbf{1 1}}+\overline{\mathbf{5 5}}+\overline{\mathbf{1 6 5}}+\mathbf{3 3 0}$ : family replication is nowhere to be seen at a fundamental level, only emerging at low energies due to spontaneous symmetry breaking.

This constitutes a strong motivation for studying viable ways of embedding the Standard Model fermions in GUT representations beyond the usual scenarios. There are other reasons: for example, the way SM fermions are embedded can have a rather curiously and dramatic effect on the unification of the three gauge couplings. To appreciate it, let us consider first the standard extrapolation of the value of these couplings to higher energies, assuming only the Standard Model fields (full lines in figure 1), which are known not to unify. Importantly, one usually compares the values of $g_{1}=\sqrt{5 / 3} g^{\prime}, g_{2}=g$ and $g_{3}=g_{s}$, with the factor $\sqrt{5 / 3}$ being directly related to the way in which fermions are presumed to be embedded. In other words, figure 1 is not just a product of what is known at low energies; it also incorporates an assumption. The assumption is that the $d^{c}$ and the $L$ fermions are contained in the representation $\overline{\mathbf{5}}$ of $S U(5)$. In particular, the hypercharge matrix 
$Y=n \operatorname{diag}(1 / 3,1 / 3,1 / 3,-1 / 2,-1 / 2)$ must have the same norm as all other $S U(5)$ generators, so equating $\operatorname{Tr}(Y Y)$ with $\operatorname{Tr}\left(T_{S U(2)}^{3} T_{S U(2)}^{3}\right)$ - where $T_{S U(2)}^{3}=\operatorname{diag}(0,0,0,1 / 2,-1 / 2)$ is the diagonal SM $S U(2)$ generator - yields $n=\sqrt{3 / 5}$, therefore $y_{\text {norm. }}=\sqrt{3 / 5} y$ and $g_{1}=\sqrt{5 / 3} g^{\prime}$.

This factor is the same for $S O(10)$ and $E_{6}$ GUTs, but different arrangements could conceivably yield $n \neq \sqrt{3 / 5}$, potentially leading to a situation where the three gauge couplings unify with no extra fields lighter than the unification scale. This is certainly possible mathematically; the question is whether or not those scenarios are associated with viable models. In fact, mathematically one can conceivably even spoil the relations $g_{2}=g$ and $g_{3}=g_{s}$. For example, an $S U(7)$ model utilizing the branching rule $\mathbf{7} \rightarrow d^{c}+L+(\mathbf{1}, \mathbf{2}, 0)$ would imply that $g_{2}=\sqrt{2} g$ (dashed line in figure 1 ). ${ }^{1}$

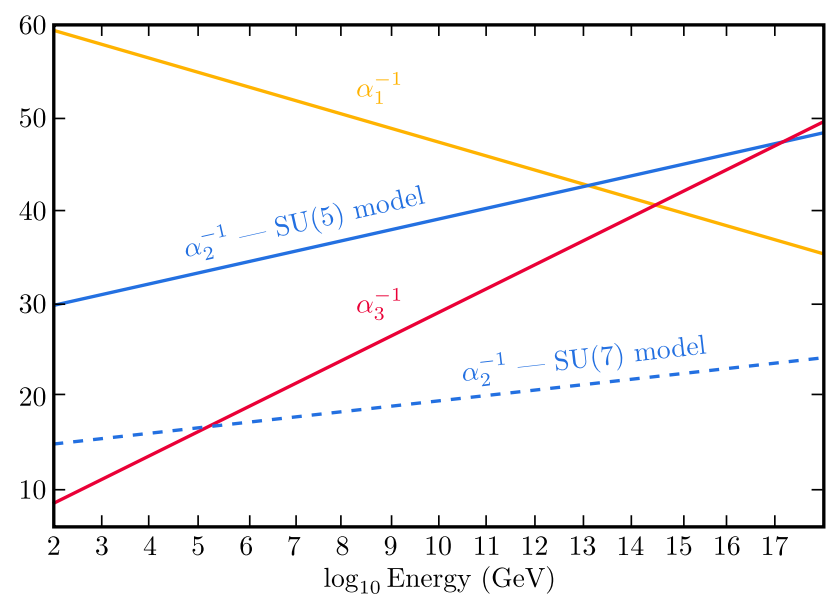

Figure 1: Bottom-up evolution of the Standard Model gauge couplings at one loop, with $\alpha_{i}^{-1} \equiv 4 \pi / g_{i}^{2}$. The usual picture (full lines) makes the assumption that fermions are embedded as in $S U(5)$. But other scenarios might spoil the relation $g_{1}=\sqrt{5 / 3} g^{\prime}$ and it is even possible that $\left(g_{2}, g_{3}\right) \neq\left(g, g_{s}\right)$ : for example an $S U(7)$ embedding might yield the dashed line.

\section{Viable fermion GUT representations}

The only fermions which can have a pure electroweak mass are most likely the Standard Model ones, so if there are more fermions they must have a mass even before electroweak symmetry is broken. These extra fermions - if they exist at all - must be vector-like. In order for this to be true, the fermion GUT representations $R+R^{\prime}+\cdots$ must decompose under $G_{S M}$ into the 3 chiral families plus a real representation (of $G_{S M}$ ) which can be reducible:

$$
R+R^{\prime}+\cdots \rightarrow 3\left(Q+u^{c}+d^{c}+L+e^{c}\right)+\text { real representation } .
$$

It might not sound like much, but this simple constraint on the fermion field content of grand unified theories turns out to be quite stringent. In reference [10] a search was made over (a)

${ }^{1}$ One might ask why is it so important to normalize all generators of the GUT group such that $\operatorname{Tr}\left(T^{a} T^{b}\right) \propto \delta^{a b}$. The reason is this: the structure constants $c^{a b c}$ appearing in the commutator relation $\left[T^{a}, T^{b}\right]=i c^{a b c} T^{c}$ control the transformation of gauge bosons under infinitesimal global transformations: $A_{\mu}^{a} \rightarrow A_{\mu}^{a}-c^{b c a} A_{\mu}^{b} \alpha^{c}$ (where $\alpha$ is the transformation parameter). And in order for this to correspond to a unitary transformation, $c^{a b c}$ must be a completely antisymmetric tensor, which in turn requires that $\operatorname{Tr}\left(T^{a} T^{b}\right) \propto \delta^{a b}$. 
different simple groups $G$, (b) different combinations of representations $R, R^{\prime}, \ldots$ of $G$ and (c) different embeddings of $G_{S M}$ in $G$ (there might be more than one). Under some reasonable assumptions, such as the nonexistence of confining interactions besides those of $S U(3)_{c}$ at very low energies, the scan showed that

- The viable simple groups $G$ are $S O(10), E_{6}$ and the $S U(N)$ 's for $N \geq 5$.

- The Standard Model group can only be embedded in one way in all these groups, except for $S U(N \geq 15)$. There is no viable alternative to the relations $g_{1}=\sqrt{5 / 3} g^{\prime}, g_{2}=g$ and $g_{3}=g_{s}$.

- Apart from trivial variations, the fermion content in $S U(5)(3 \times \overline{\mathbf{5}}+3 \times \mathbf{1 0})$ and $E_{6}(3 \times \mathbf{2 7})$ GUTs is unique. Furthermore, for all practical purposes so is the one in an $S O(10)$ model $(3 \times 16)$. On the other hand, for $S U(N \geq 6)$ there are various ways of embeddings the SM fermions, and crucially family replication is not a requirement.

- The only non-trivial case where all fermions can be embedded in a single representation ${ }^{2}$ is by using the $\mathbf{1 7 1}$ representation of $S U(19)$. This possibility was also mentioned in [11].

The main features of an $S U(19)$ model exploring this last idea were considered in [12], which provides a glimpse of how flavor might arise from a fundamental theory which has no family replication. I used the word glimpse because, as we shall see below, in order to compute the Standard Model Yukawa matrices $Y_{U}, Y_{D}$ and $Y_{E}$ at the electroweak scale one needs to calculate the ratio of several vacuum expectations values (VEVs), which is a daunting task still to be addressed.

\section{A model for flavorgenesis}

With a single fermion representation $\mathbf{1 7 1}$ of the $S U(19)$ gauge group, the fermion-fermionscalar interactions are controlled by a singlet number $y$ for each scalar irreducible representation $\Phi$,

$$
\mathscr{L}_{\text {Yukawa }}=y 171 \cdot 171 \cdot \Phi .
$$

There are two possible quantum numbers for $\Phi$ : it could either transform as $\overline{\mathbf{3 8 7 6}}$ or $\overline{\mathbf{1 0 8 3 0}}$ and reference [12] considered the first possibility. But how might the entries of $Y_{U}, Y_{D}$ and $Y_{E}$ be generated from just a single number? The answer is the following. The scalar $\Phi$ contains several components which transform as $S \equiv(\mathbf{1}, \mathbf{1}, 0), H \equiv(\mathbf{1}, \mathbf{2}, 1 / 2)$ and $\widetilde{H} \equiv(\mathbf{1}, \mathbf{2},-1 / 2)$ under the Standard Model gauge group, and it is the ratio of their VEVs that control the entries of $Y_{U}, Y_{D}$ and $Y_{E}$. In fact, the coupling constant $y$ in equation (3) merely acts as an overall normalization factor for these three matrices.

In order to go further, we need to break down the single $S U(19)$-invariant term above into several pieces which are only invariant under the $G_{S M}$ subgroup. Fortunately, only a few of them are relevant at low energies. Among others, we find the following $G_{S M}$ representations inside the larger $S U(19)$ ones:

$$
\begin{aligned}
& 171 \ni Q_{i}, Q^{c}, u_{i}^{c}, u, d_{i}^{c}, d_{5}^{c}, d_{1}, d_{2}, L_{i}^{c}, L_{5}^{c}, L_{1}, L_{2}, e_{i}^{c}, e, N_{i j}^{c}, \\
& \overline{\mathbf{3 8 7 6}} \ni S_{L}, S_{D L}, S_{D}, S_{U D}^{i}, S_{Q D L}^{i}, S_{E L}^{i}, S_{N}, H_{Q N}^{i j}, H_{N, i}, \widetilde{H}_{D E}^{i}, \widetilde{H}_{D}^{i j}, \widetilde{H}_{E}^{i j} .
\end{aligned}
$$

${ }^{2}$ This can also trivially be achieved with the fundamental representation of $S U(45)$, or even bigger special unitary groups if we were to consider extra vector-like fermions. 
The quantum numbers associated to the symbols appearing here have been mentioned before except those of $Q^{c}, u, d, L^{c}$ and $e$ which are the vector-like partners of the Standard Model fermion representations. On the other hand the indices $i$ and $j$ indicate that a field transforms under an extra $S U(4)_{F}$ found inside $S U(19)$, and which commutes with $G_{S M}$. For example, $Q_{i}$ is a quadruplet of this group (lower index), the scalar $S_{E L}^{i}$ is an anti-quadruplet (upper index) and $Q^{c}$ is a singlet (no index). These indices must be antisymmetrized, therefore $N_{i j}^{c}$ transforms as the antisymmetric product of two quadruplets of $S U(4)_{F}$ - a sextet.

We then see that there are $4 Q^{\prime}$ 's and $1 Q^{c} ; 5 d^{c}$ 's and $2 d$ 's, and so on. All in all, there is always an excess of 3 copies of a fermion representation $X$ over its vector-like partner $X^{c}$ which ensures that at low energies only the Standard Model fermions are observed. The precise composition of these light fermions is controlled by the VEVs of the $S$ scalars: indeed we find that the $S U(19)$-invariant term in equation (3) contains the $G_{S M}$-invariant pieces

$$
y^{-1} \mathscr{L}_{\text {Yukawa }} \supset Q_{i} \mathbf{M}_{\mathbf{Q}} Q^{c}+u_{i}^{c} \mathbf{M}_{\mathbf{U}} u+e_{i}^{c} \mathbf{M}_{\mathbf{E}} e+N_{i j}^{c} \mathbf{M}_{\mathbf{N}} N_{k l}^{c}+\left(\begin{array}{c}
d_{i}^{c} \\
d_{5}^{c}
\end{array}\right)^{T} \mathbf{M}_{\mathbf{D}}\left(\begin{array}{c}
d_{1} \\
d_{2}
\end{array}\right)+\left(\begin{array}{c}
L_{i} \\
L_{5}
\end{array}\right)^{T} \mathbf{M}_{\mathbf{L}}\left(\begin{array}{c}
L_{1}^{c} \\
L_{2}^{c}
\end{array}\right)
$$

where

$$
\begin{gathered}
\mathbf{M}_{\mathbf{Q}}=\frac{1}{3} S_{Q D L}^{i}, \mathbf{M}_{\mathbf{U}}=\frac{\sqrt{2}}{3} S_{U D}^{i}, \mathbf{M}_{\mathbf{E}}=\sqrt{\frac{2}{3}} S_{E L}^{i}, \mathbf{M}_{\mathbf{N}}=\frac{1}{4} \sqrt{\frac{2}{3}} \epsilon_{i j k l} S_{N}, \\
\mathbf{M}_{\mathbf{D}}=\frac{\sqrt{2}}{3}\left(\begin{array}{cc}
-S_{Q D L}^{i} & \sqrt{2} S_{U D}^{i} \\
-S_{D L} & \sqrt{2} S_{D}
\end{array}\right), \mathbf{M}_{\mathbf{L}}=\frac{1}{\sqrt{3}}\left(\begin{array}{cc}
-S_{Q D L}^{i} & \sqrt{2} S_{E L}^{i} \\
-\frac{2}{\sqrt{3}} S_{L} & S_{D L}
\end{array}\right) .
\end{gathered}
$$

For example, if $\left\langle S_{Q D L}\right\rangle=(0,1,0,0)^{T}$ and $\left\langle S_{U D}\right\rangle=(1,0,0,0)^{T}$ then the fermions $Q_{1,3,4}$ are massless until the electroweak symmetry is broken, and so are $u_{2,3,4}^{c}$. Furthermore, the right-hand side of equation (3) also contains the following interactions with $H$ and $\widetilde{H}$ fields:

$$
y^{-1} \mathscr{L}_{\text {Yukawa }} \supset Q_{i} \mathbf{Y}_{\mathbf{U}} u_{j}^{c}+Q_{i} \mathbf{Y}_{\mathbf{D}}\left(\begin{array}{c}
d_{j}^{c} \\
d_{5}^{c}
\end{array}\right)+\left(\begin{array}{c}
L_{i} \\
L_{5}
\end{array}\right)^{T} \mathbf{Y}_{\mathbf{E}} e_{i}^{c}+\left(\begin{array}{c}
L_{i} \\
L_{5}
\end{array}\right)^{T} \mathbf{Y}_{\mathbf{N}} N_{j k}^{c}
$$

where $\mathbf{Y}_{\mathbf{U}}=-2 / 3 H_{Q N}^{i j}$ and formulas for the remaining $\mathbf{Y}_{\mathbf{X}}$ can be found in [12]. Recall that the two upper indices mean that $H_{Q N}^{i j}$ is a sextet of the $S U(4)_{F}$ flavor group and therefore it can be written as an anti-symmetric 4 by 4 matrix. The Standard Model Higgs boson $H_{S M}$ must be a combination of all the $H$ and $\widetilde{H}$ fields, so we can write that $H_{Q N}^{i j}=\Lambda_{Q N}^{i j} H+\cdots$ where $\Lambda_{Q N}$ is some anti-symmetric matrix of coefficients. With this notation and taking $\left\langle S_{Q D L}\right\rangle=(0,1,0,0)^{T}$ and $\left\langle S_{U D}^{i}\right\rangle=(1,0,0,0)^{T}$ as an example, the $\mathrm{SM} Y_{U}$ matrix would be given by the expression

$$
Y_{U}=-\frac{2}{3} y\left(\begin{array}{ccc}
\Lambda_{Q N}^{12} & \Lambda_{Q N}^{13} & \Lambda_{Q N}^{14} \\
-\Lambda_{Q N}^{23} & 0 & \Lambda_{Q N}^{34} \\
-\Lambda_{Q N}^{24} & -\Lambda_{Q N}^{34} & 0
\end{array}\right)
$$

Similar calculations can be done for the remaining Standard Model Yukawa matrices, as well as for the neutrino masses $1 / 2\left(m_{v}\right)_{\alpha \beta} v_{L, \alpha} v_{L, \beta}$. In this way, flavor might be generated effectively at low energies from a fundamental theory which is flavorless. Still, in order to confront this model 
with the observed fermion masses and mixing data, it would be necessary to also examine the VEVs which minimize the scalar potential.

\section{Conclusions}

Grand unified theories, which have been proposed and studied for more than four decades, provide a potential explanation for the Standard Model quantum numbers, as well as the values of the three gauge couplings. However, standard GUTs do not explain the phenomena of fermion family replication. In this work I discussed how non-standard GUTs might do so.

\section{Acknowledgments}

I am grateful to Andreas Ekstedt and Michal Malinský for their collaboration in the paper [12], on which the present work is partially based. I acknowledge the financial support from the Grant Agency of the Czech Republic (GAČR) through contract number 20-17490S and from the Charles University Research Center UNCE/SCI/013.

\section{References}

[1] H. Georgi and S. Glashow, Unity of all elementary particle forces, Phys. Rev. Lett. 32 (1974) 438.

[2] H. Georgi, The state of the art: gauge theories, AIP Conf. Proc. 23 (1975) 575.

[3] H. Fritzsch and P. Minkowski, Unified interactions of leptons and hadrons, Annals Phys. 93 (1975) 193.

[4] F. Gürsey, P. Ramond and P. Sikivie, A Universal gauge theory model based on $E_{6}$, Phys. Lett. B 60 (1976) 177.

[5] M. Singer, J. Valle and J. Schechter, Canonical neutral current predictions from the weak electromagnetic gauge group $S U(3) \times U(1)$, Phys. Rev. D 22 (1980) 738.

[6] F. Pisano and V. Pleitez, An $S U(3) \times U(1)$ model for electroweak interactions, Phys. Rev. $D$ 46 (1992) 410 [hep-ph/9206242].

[7] P. Frampton, Chiral dilepton model and the flavor question, Phys. Rev. Lett. 69 (1992) 2889.

[8] R.M. Fonseca and M. Hirsch, A flipped 331 model, JHEP 08 (2016) 003 [1606.01109].

[9] H. Georgi, Towards a grand unified theory of flavor, Nucl. Phys. B 156 (1979) 126.

[10] R.M. Fonseca, On the chirality of the SM and the fermion content of GUTs, Nucl. Phys. B 897 (2015) 757 [1504.03695].

[11] N. Yamatsu, Family unification in special grand unification, PTEP 2018 (2018) 091B01 [1807.10855].

[12] A. Ekstedt, R.M. Fonseca and M. Malinský, Flavorgenesis, 2009.03909. 